\title{
Atitudes Éticas dos Profissionais de Saúde dos Cuidados de Saúde Primários Perante os Direitos que Visam a Autonomia do Paciente
}

José Augusto Simões*, José Carlos Martins**

\section{Pontos-chave:}

$\mathrm{Na}$ atualidade não é simples falar de moral, embora a ética esteja na moda, contudo parece que poucos acreditam que seja uma disciplina importante para a vida em geral.

Considerando que existem poucos estudos sobre as bases que influenciam as decisões éticas dos profissionais de saúde, o objetivo deste estudo foi analisar as atitudes éticas dos profissionais de saúde nas relações com os pacientes.

\section{Resumo}

Objetivo: Considerando que existem poucos estudos sobre as bases que influenciam as decisões éticas dos profissionais de saúde, o objetivo deste estudo foi analisar as atitudes éticas dos profissionais de saúde nas relações com os pacientes.

Métodos: Estudo transversal quantitativo, que envolveu médicos e enfermeiros dos centros de saúde da região centro de Portugal. Uma escala, validada, para avaliar as atitudes éticas dos profissionais de saúde, foi aplicada a uma amostra de médicos e enfermeiros de cuidados de saúde primários através de questionário auto preenchido. Software: SPSS 15.0. Testes estatísticos: Mann-Whitney and Kruskal-Wallis.

Resultados: Foram inquiridos 163 profissionais de saúde, 77 médicos e 86 enfermeiros, $74,2 \%$ do sexo feminino, com idade média de 45,8 anos. As atitudes éticas dos profissionais de saúde não parecem ser influenciadas pelo género, idade ou anos de atividade. No entanto, os médicos em relação aos enfermeiros parecem ter atitudes éticas mais firmes perante os direitos que visam a autonomia do paciente, assim como os profissionais de saúde que trabalham nas sub-regiões de saúde de Viseu e Aveiro e em área urbana.

\begin{abstract}
Aim: whereas there are few studies on the basis that influence ethical decisions of health professionals, the aim of this study was to analyze the ethical attitudes of health professionals in dealing with patients.

Methods: A quantitative cross-sectional study involving doctors and nurses from the health centers of the central region of Portugal. A validated scale to assess the ethical attitudes of health professionals was applied to a sample of physicians and nurses in primary health care physicians and primary health care nurses through a self filled. Software: SPSS 15.0. Statistical tests: Mann-Whitney and Kruskal-Wallis.
\end{abstract}

Results: We surveyed 163 health professionals, 77 doctors and 86 nurses, $74.2 \%$ female, mean age of 45.8 years. Ethical attitudes of health professionals do not seem to be influenced by gender, age or years of activity. However, doctors in relation to nurses seem to have firmer ethical attitudes towards the rights aimed at the patient's autonomy, as well as health professionals working in the health sub-region of Viseu and Aveiro and in urban areas.

Discussion: The doctors and nurses in primary health care are concerned with preserving the ethics of their

\footnotetext{
Médico de Família na USF Marquês de Marialva, ACeS Baixo Mondego.

${ }^{* *}$ Enfermeiro. Professor Coordenador na Escola Superior de Enfermagem de Coimbra.
} 
Discussão: Os médicos e enfermeiros dos cuidados de saúde primários preocupam-se com a preservação da eticidade das suas atitudes para com os pacientes. São necessários mais estudos sobre a interface da bioética com os cuidados de saúde primários.

Palavras-chave: Cuidados de Saúde Primários. Escala de Atitudes. Bioética. Médicos. Enfermeiros.

\section{Introdução}

Na atualidade não é simples falar de moral, embora a ética esteja na moda, contudo parece que poucos acreditam que seja uma disciplina importante para a vida em geral. Ética e moral remetem-nos para os costumes e ajudam-nos a forjar um carácter bom e a confrontar a vida de um modo mais humano de forma que, no final, possamos ser mais felizes ${ }^{1}$.

A ética é uma disciplina de carácter prático que pretende guiar a ação humana com um sentido racional. Embora vivamos num tempo de certo relativismo moral e se defenda a convicção que não é possível discutir na ética coisas que são completamente subjetivas e privadas ${ }^{2}$, a própria sociedade pede a imposição de limites práticos e reconhece que em certas ocasiões nem tudo vale, e nem tudo vale por igual ${ }^{3,4}$.

O campo da saúde é cada vez mais influenciado pelos avanços científicos e tecnológicos que provocaram uma crescente complexidade da medicina. A utilização destas novas tecnologias deve ser analisada sob um ponto de vista ético, assim como as mudanças que aconteceram nas relações entre os profissionais da saúde e os utentes dos serviços de saúde nas últimas décadas. A bioética cresceu ao mesmo tempo que os problemas com o ambiente e as ciências da vida (medicina, enfermagem, biologia, ecologia,...) e esboçou alguns princípios gerais para se poder decidir se tudo o que é tecnicamente possível é eticamente correto e bom ${ }^{5,6}$.

A bioética está implícita na atividade diária, mas de um modo inconsciente. Em geral é observada uma rejeição pelos tópicos que insinuam a autocrítica. Os conflitos éticos derivam fundamentalmente da relação entre o profissional de saúde e o doente, pontuada pela família, principalmente ao dar informação. Os princípios bioéticos consideraram como muito importante os direitos do doente à informação e ao consentimento informado?.

Designa-se por consentimento informado o acordo livre e esclarecido que um ser humano presta em relação attitudes towards patients. Further studies are needed on the bioethics interface with primary health care.

Keywords: Ethics, Primary Health Care, Attitudes Scale, Family Practice, Nursing.

a quaisquer atos médicos a serem praticados na sua pessoa. O consentimento informado é uma das consequências do primeiro princípio da Bioética: a autonomia de todo e qualquer ser humano, baseado na dignidade da pessoa e no direito que ela tem à sua autorrealização individual ${ }^{8}$.

Está a tornar-se universalmente aceite que é dever do médico fornecer a informação, o mais completa possível, a respeito de todo o procedimento que deva ser empreendido, e esta informação deve incluir consequências possíveis e o prognóstico ou a esperança provável de sucesso. No entanto, não há acordo sobre se a informação e o consentimento dados em formulários mais ou menos desenvolvidos podem ser considerado consentimento informado e esclarecido ${ }^{9}$.

A pesquisa de estudos de atitudes éticas dos profissionais de saúde permitiu identificar a Escala de atitudes perante os direitos à informação e ao consentimento, elaborada e validada por José Carlos Martins ${ }^{10}$, uma escala tipo Likert com 31 itens e cinco possibilidades de resposta em cada item, que pareceu ser adequada para avaliar as atitudes éticas dos profissionais de saúde dos cuidados de saúde primários.

Neste sentido, e após já terem sido estudadas as atitudes de doentes oncológicos perante os direitos à informação e ao consentimento ${ }^{10}$, as atitudes dos enfermeiros do Centro Regional de Oncologia de Coimbra ${ }^{11}$ e as atitudes de médicos de família ${ }^{12}$ perante esses mesmos direitos, pareceu-nos pertinente levar a cabo um estudo das atitudes dos profissionais de saúde dos cuidados de saúde primários perante os direitos dos doentes à informação e ao consentimento. Desenvolvemos uma investigação descritiva e correlacional, centrada nos seguintes objetivos:

- Analisar o quadro de atitudes dos profissionais de saúde dos cuidados de saúde primários, perante os direitos que visam a autonomia do doente; 
- Analisar as relações entre a idade, o género, a profissão, o número de anos de profissão, a sub-região e a área de trabalho dos profissionais de saúde dos cuidados de saúde primários e a atitude perante os direitos que traduzem a autonomia;

- Comparar as perceções e atitudes dos médicos com as opiniões e atitudes dos enfermeiros.

Formularam-se as seguintes hipóteses:

H1. As atitudes éticas dos profissionais de saúde não são influenciáveis pela profissão (médico ou enfermeiro).

H2. As atitudes éticas dos profissionais de saúde não são influenciáveis pelo género (masculino ou feminino).

H3. As atitudes éticas dos profissionais de saúde não são influenciáveis pela idade.

H4. As atitudes éticas dos profissionais de saúde não são influenciáveis pelo número de anos de profissão.

H5. As atitudes éticas dos profissionais de saúde não são influenciáveis pela sub-região em que trabaIham (Aveiro, Castelo Branco, Coimbra, Guarda, Leiria, Viseu).

H6. As atitudes éticas dos profissionais de saúde não são influenciáveis pela área geográfica em que trabalham (urbana, rural, semiurbano).

\section{Métodos}

Realizou-se um estudo observacional, transversal, descritivo, e com componente analítica, através da aplicação de um questionário, construído para o estudo, na área geográfica de intervenção da Administração Regional de Saúde do Centro (ARS-C).

O universo estudado foram os médicos de família e os enfermeiros a trabalhar nos centros de saúde (CS) da ARS-C. Devido ao número de profissionais a estudar ser de 1660 médicos e 1659 enfermeiros ${ }^{13}$ e à sua dispersão geográfica, houve necessidade de obter uma amostra que fosse representativa de toda a região. Por não haver indicadores primários, nem dados de estudos anteriores que permitissem calcular um número significativo, optou-se por uma amostragem de agrupamento ${ }^{14}$ com base geográfica no distrito, que correspondia a cada sub-região de saúde (SRS). Assim, foram definidas as amostras das populações a estudar: médicos de família (especialistas de medicina geral e familiar e clínicos gerais com listas de utentes) e enfermeiros (especialistas e não especialistas) a trabalhar em $50 \%$ dos CS de cada SRS da área da ARS-C. Os centros de saúde foram ordenados alfabeticamente, por sub-região de saúde, seguindo-se um sorteio, por números aleatórios, dos CS até perfazer os 50\%. A aleatorização de centros de saúde acabou por determinar a seleção de CS com um total de 936 (56\%) dos médicos de família e 816 (49\%) dos enfermeiros da área geográfica de intervenção da Administração Regional de Saúde do Centro.

Antes de se iniciar a aplicação do questionário foi pedido à Comissão de Ética para a Saúde do Centro de Saúde de São João, do Porto, a elaboração de um parecer para a realização do estudo; e ao Conselho Diretivo da Administração Regional de Saúde do Centro, a necessária autorização para a realização do mesmo.

Os diretores dos centros de saúde aleatorizados foram contatados, pessoalmente ou pelo correio, pedindo-se-Ihes colaboração na distribuição dos questionários junto de todos os médicos de família e enfermeiros em serviço no seu CS, bem como na recolha e sua devolução ao investigador.

O questionário utilizado neste estudo era composto por duas partes. A primeira incluiu seis questões sobre os aspetos sócio profissionais de caraterização do inquirido: idade, sexo, profissão, número de anos de profissão, sub-região e área de trabalho. A segunda parte incluiu a Escala de atitudes perante os direitos à informação e ao consentimento, elaborada e validada por José Carlos Martins ${ }^{15}$, escala tipo Likert com 31 itens.

\section{Resultados}

Só foram recebidos 163 questionários devidamente preenchidos, o que representou uma muito baixa taxa de resposta $(9,3 \%)$ dos profissionais de saúde dos centros de saúde aleatorizados na Região Centro.

A amostra em estudo ficou constituída por 121 de indivíduos do sexo feminino e 42 do sexo masculino, o que representa $74,2 \%$ e $25,8 \%$ respetivamente.

As principais características sócio profissionais da amostra são apresentadas no quadro I.

A média de idades é de 45,8 anos e a mediana situa-se nos 48 anos. 0 desvio padrão é de 9,09 anos. 0 indivíduo mais novo tinha 23 anos e o mais idoso 61 anos. 
Por género, a média de idades para o feminino é de 44,5 anos e a mediana situa-se nos 44 anos. 0 desvio padrão é de 9,12 anos. A mulher mais nova tinha 23 anos e a mais idosa 61 anos. A média de idades para o masculino é de 49,9 anos e a mediana situa-se nos 52 anos. 0 desvio padrão é de 7,80 anos. 0 homem mais novo tinha 23 anos e o mais idoso 58 anos.

Quando agrupados por grupos etários, verifica-se que predominam os grupos etários 50-59 anos (44,2\%) e 40-49 anos (28,2\%). Os grupos menos numerosos são o dos 20-29 anos (3,7\%) e dos 60-69 anos (1,2\%).

Profissionalmente, verifica-se que a amostra é constituída por 77 médicos (47,2\%) e por 86 enfermeiros $(52,8 \%)$.

Por profissões, a média de idades para os médicos é de 50,8 anos e a mediana situa-se nos 52 anos. 0 desvio padrão é de 6,78 anos. 0 indivíduo mais novo tinha 30 anos e o mais idoso 61 anos. A média de idades para os enfermeiros é de 41,4 anos e a mediana situa-se nos 41 anos. 0 desvio padrão é de 8,56 anos. 0 indivíduo mais novo tinha 23 anos e o mais idoso 61 anos.

Quanto à sub-região a que pertencem, verifica-se que 31 indivíduos da amostra pertencem a Aveiro (19,0\%), 49 a Castelo Branco (30,1\%), 48 a Coimbra (29,4\%), 20 a Leiria (12,3\%), 10 à Guarda (6,1\%) e por fim, só 5 indivíduos pertencem a Viseu (3,1\%).

Quanto à área de trabalho, 83 indivíduos consideram que trabalham em meio urbano (50,9\%), 26 em meio rural $(16,0 \%)$ e 54 consideram que trabalham num meio semiurbano $(33,1 \%)$.

Quadro I: Distribuição dos elementos da amostra de acordo com as características socioprofissionais

\begin{tabular}{lcc} 
Variáveis & $\mathbf{N}^{\circ}$ & $\%$ \\
\hline Género & & \\
\hline Feminino & 121 & 74,2 \\
Masculino & 42 & 25,2 \\
\hline Idade & & \\
\hline $20-29$ anos & 6 & 3,7 \\
$30-39$ anos & 37 & 22,7 \\
$40-49$ anos & 46 & 28,2 \\
$50-59$ anos & 72 & 44,2 \\
$60-69$ anos & 2 & 1,2
\end{tabular}

\begin{tabular}{lcc}
\hline Profissão & 77 & 47,2 \\
\hline Médico & 86 & 52,8 \\
\hline Enfermeiro & & \\
\hline Sub-Região & 31 & 19,0 \\
\hline Aveiro & 49 & 30,1 \\
Castelo Branco & 48 & 29,4 \\
Coimbra & 10 & 6,1 \\
Guarda & 20 & 12,3 \\
Leiria & 5 & 3,1 \\
Viseu & & \\
\hline Área de Trabalho & 83 & 50,9 \\
\hline Urbana & 26 & 16,0 \\
Rural & 54 & 33,1 \\
Semiurbana &
\end{tabular}

De acordo com os dados obtidos, pode-se constatar que os respondentes apresentam uma atitude de concordância em relação aos itens inquiridos com uma média de resposta de 3.99, com um mínimo de 3.07 e um máximo de 4.80 (quadro III). As respostas podiam variar numa escala de Likert, entre um e cinco, representando o valor "um" a discordância completa e o valor "cinco" a concordância completa (em relação ao item).

Quadro II: Estatística descritiva relativa às atitudes dos profissionais de saúde perante os perante os direitos à informação e ao consentimento dos pacientes

\begin{tabular}{c|c} 
& Escala \\
\hline No de itens $^{\circ}$ & 31 \\
\hline Média & 3.99 \\
\hline Mediana & 3.93 \\
\hline Desvio Padrão & 0.36 \\
\hline Variância & 0.13 \\
\hline Mínimo & 3.07 \\
\hline Máximo & 4.80
\end{tabular}

Testando as hipóteses formuladas:

H1. As atitudes éticas dos profissionais de saúde não são influenciáveis pela profissão (médico ou enfermeiro). 
Verificou-se que a média das pontuações foi superior no grupo dos médicos (quadro III), como a variável da pontuação da escala não segue uma distribuição normal, nos grupos profissionais, teve que se aplicar o teste não paramétrico Mann-Whitney, e estatisticamente rejeitase a hipótese nula ( $p<0.5)$. Pode assim concluir-se que as atitudes perante problemas éticos nas relações dos profissionais de saúde com os pacientes e suas famílias são influenciáveis pela profissão. Considerando como mais firme a obtenção de pontuações superiores na avaliação feita através da aplicação da escala, pode considerar-se como mais firmas as atitudes éticas dos médicos face aos enfermeiros perante os direitos à informação e ao consentimento dos pacientes.

Quadro III: Atitudes éticas dos inquiridos segundo a profissão

\begin{tabular}{|c|c|c|}
\hline & $\begin{array}{c}\text { Média da pontuação } \\
\text { na escala A }\end{array}$ & $\begin{array}{c}\text { Teste } \\
\text { Mann-Whitney }\end{array}$ \\
\hline Médicos $(n=77)$ & 4.02 & $p=0.031$ \\
Enfermeiros $(n=86)$ & 3.96 & \\
\hline
\end{tabular}

H2. As atitudes éticas dos profissionais de saúde não são influenciáveis pelo género (masculino ou feminino).

Verificou-se que a média das pontuações para a escala foram iguais nos dois géneros $=3.99$. Não se rejeitando esta hipótese nula. Conclui-se assim que as atitudes dos profissionais de saúde perante os direitos à informação e ao consentimento dos pacientes não são influenciáveis pelo género.

H3. As atitudes éticas dos profissionais de saúde não são influenciáveis pela idade.

Como nenhuma das duas variáveis segue uma distribuição normal, aplicou-se o coeficiente de Spearman que tem um valor de teste de 0.059 (correlação positiva fraca), associado a um valor p de 0.257 (Gráfico I). Não se rejeitando esta hipótese nula. Conclui-se assim que as atitudes dos profissionais de saúde perante os direitos à informação e ao consentimento dos pacientes não são influenciáveis pela idade.
Gráfico I: Atitudes éticas dos inquiridos segundo a idade

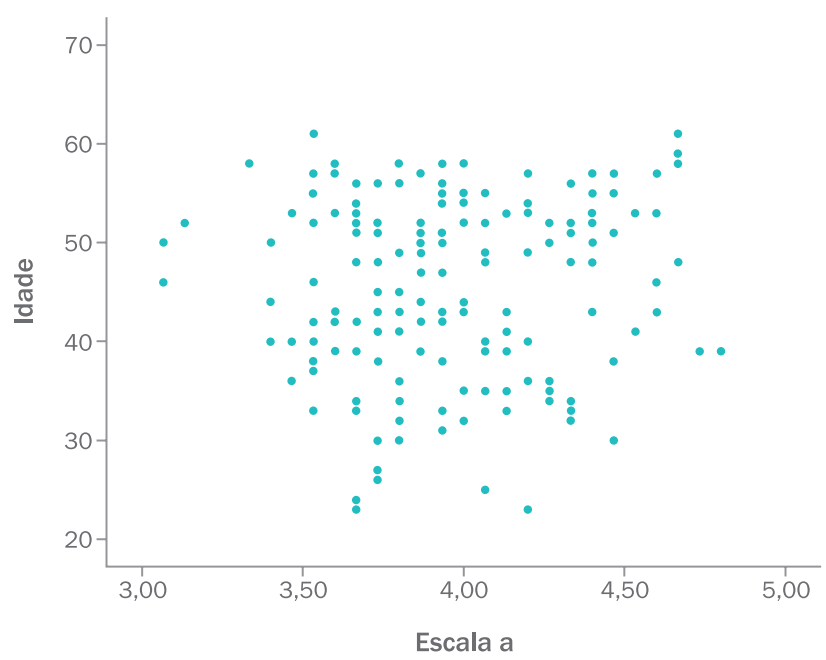

H4. As atitudes éticas dos profissionais de saúde não são influenciáveis pelo número de anos de profissão.

Como nenhuma das duas variáveis segue uma distribuição normal, aplicou-se o coeficiente de Spearman que tem um valor de teste de 0.111 (correlação positiva fraca), associado a um valor p de 0.033 (Gráfico II). Não se rejeitando esta hipótese nula. Conclui-se assim que as atitudes dos profissionais de saúde perante os direitos à informação e ao consentimento dos pacientes não são influenciáveis pelo número de anos de profissão.

Gráfico II: Atitudes éticas dos inquiridos segundo o número de anos de profissão

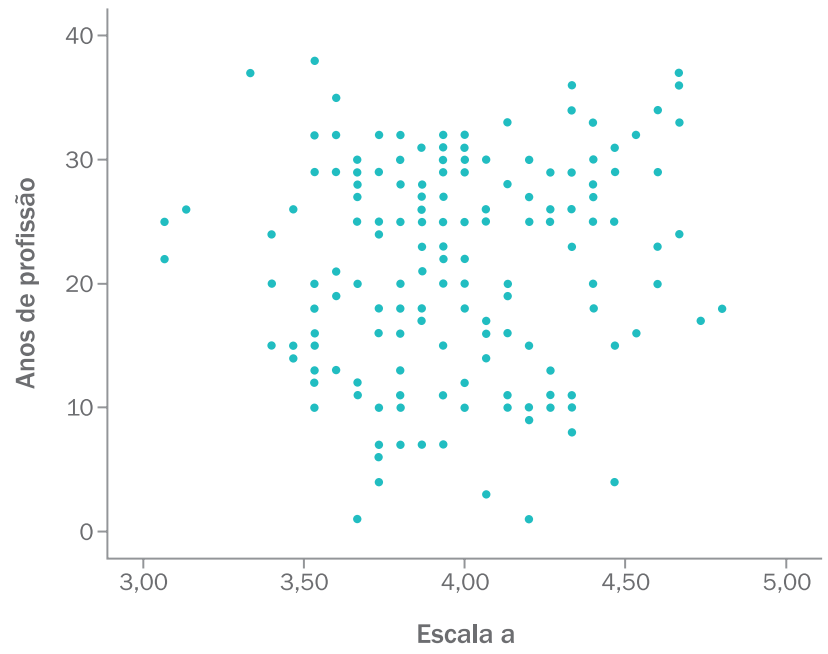


H5. As atitudes éticas dos profissionais de saúde não são influenciáveis pela sub-região em que trabaIham (Aveiro, Castelo Branco, Coimbra, Guarda, Leiria, Viseu).

Verificou-se que a média das pontuações foi superior no grupo dos profissionais que trabalham na sub-região de Viseu e inferior nos que trabalham na sub-região de Castelo Branco (quadro IV), como a variável da pontuação da escala não segue uma distribuição normal em todas as subamostras, pelo que aplicou-se o teste não paramétrico de Kruskal-Wallis, e estatisticamente rejeita-se a hipótese nula ( $p<0.001$ ). Pode assim concluir-se que as atitudes dos profissionais de saúde perante os direitos à informação e ao consentimento dos pacientes são influenciáveis pela sub-região em que trabalham. Considerando como mais firme a obtenção de pontuações superiores na avaliação feita através da aplicação da Escala de atitudes perante os direitos à informação e ao consentimento, pode considerar-se como mais firmas as atitudes éticas nas sub-regiões de saúde de Viseu e Aveiro e menos firmes nas sub-regiões de saúde de Leiria e Castelo Branco.

Quadro IV: Atitudes éticas dos inquiridos em função da sub-região de saúde a que pertencem

\begin{tabular}{l|c|c|} 
& $\begin{array}{c}\text { Média da pontuação } \\
\text { na escala A }\end{array}$ & $\begin{array}{c}\text { Teste } \\
\text { Kruskal-Wallis }\end{array}$ \\
\hline Aveiro & 4.03 & \\
Castelo Branco & 3.88 & \\
Coimbra & 4.01 & $\mathrm{p}<0.001$ \\
Guarda & 3.99 & \\
Leiria & 3.92 & \\
Viseu & 4.29 & \\
\hline
\end{tabular}

H6. As atitudes éticas dos profissionais de saúde não são influenciáveis pela área geográfica em que trabalham (urbana, rural, semirrural/urbano).

Verificou-se que a média das pontuações foi superior no grupo dos profissionais que trabalham em zona urbana (quadro V), como a variável da pontuação da escala não segue uma distribuição normal em todas as subamostras, pelo que aplicou-se o teste não paramétrico de Kruskal-Wallis, e estatisticamente rejeita-se a hipótese nula ( $p<0.001$ ). Pode assim concluir-se que as atitudes perante problemas éticos nas relações dos profissionais de saúde com os pacientes e suas famílias são influenciáveis pela área geográfica em que trabalham. Considerando como mais firme a obtenção de pontuações superiores na avaliação feita através da aplicação da Escala de atitudes perante os direitos à informação e ao consentimento, pode considerar-se como mais firmas as atitudes éticas dos profissionais que declararam trabalhar em área urbana.

Quadro V: Atitudes éticas dos inquiridos em função da área geográfica em que trabalham

\begin{tabular}{l|c|c} 
Urbana & $\begin{array}{c}\text { Média da pontuação } \\
\text { na escala }\end{array}$ & $\begin{array}{c}\text { Teste } \\
\text { Kruskal-Wallis }\end{array}$ \\
Rural & 4.06 & \\
Semiurbana & 3.92 & $\mathrm{p}<0.001$ \\
\hline
\end{tabular}

\section{Discussão}

Em termos de distribuição por sexo verifica-se que $74,2 \%$ dos respondentes ao questionário são do género feminino, o que está de acordo com a informação de que os efetivos da Saúde em Portugal são predominantemente mulheres ${ }^{16}$ e de que está a ocorrer uma feminização da medicina ${ }^{17}$.

Outro dado que se pode considerar de acordo com o esperado é o facto de predominar o grupo etário dos 50-59 anos, com 44,2\% dos indivíduos. Este facto confirma o problema de carência de médicos de família a nível dos cuidados de saúde primários, por a curto prazo um grande número destes profissionais ir entrar na idade da reforma ${ }^{18}$. Aliás, a média de idades para os MF neste estudo é de 50,8 anos e a mediana situa-se nos 52 anos, enquanto a média de idades para os enfermeiros é de 41,4 anos e a mediana situa-se nos 41 anos.

Por sub-região verifica-se que pertencem a Aveiro 19,0\% dos respondentes ao questionário, 30,1\% a Castelo Branco, 29,4\% a Coimbra, 6,1\% à Guarda, 12,3\% a Leiria e só $3,1 \%$ a Viseu. Em relação à aleatorização dos CS verificase que percentualmente as sub-regiões de Castelo Branco e Coimbra estão sobre representadas nesta amostra, Aveiro e Leiria estão devidamente representadas, e Guarda e Viseu estão sub-representadas. Este facto ocorreu por ter havido uma boa participação dos profissionais dos centros de saúde de Castelo Branco e da Covilhã, e por o investigador trabalhar na sub-região de saúde de Coimbra, pelo que teve mais oportunidades para motivar os profissionais desta sub-região para o estudo. 
De acordo com os dados obtidos e descritos nos resultados, verifica-se que os inquiridos apresentam uma atitude de concordância face aos itens da escala, apresentando uma média de respostas no global da escala de 3.99, com um mínimo de 3.07 e um máximo de 4.80.

As médias das pontuações relativas aos médicos (4.02) são superiores às médias dos enfermeiros (3.96) e estatisticamente significativas ( $p=0.031$ ), levando assim a concluir que as atitudes éticas dos profissionais de saúde são influenciáveis pela profissão (médico ou enfermeiro).

Na literatura consultada encontraram-se estudos que apontam no sentido de que existem diferenças de atitude ética entre os enfermeiros e os médicos ${ }^{19,20,21,22,23,24,25}$ outros estudos que referem que não existem diferenças ${ }^{26,27,28,29,30}$ e ainda outros estudos que referem que não há diferenças no número de problemas identificados entre médicos e enfermeiros, mas que há diferenças no tipo de problemas identificados, ou seja estas diferenças não ocorrem em virtude de haver diferença no compromisso ou na abordagem ética mas, em função da estrutura hierárquica da organização assistencial e dos papéis de médicos e enfermeiros como prestadores de cuidados de saúde ${ }^{31,32}$.

Também em Portugal se antecipa que apesar das divergências existentes entre estas duas profissões, o ensino da bioética tenderá a esbater progressivamente os diferentes backgrounds existentes na medicina e na enfermagem ${ }^{33}$. Em larga medida porque independentemente da corrente ética predominante, e das tradições profissionais envolvidas, o referencial axiológico da bioética é a eminente dignidade da pessoa humana e a sua responsabilidade substantiva com os outros membros da comunidade moral no quadro de uma verdadeira solidariedade ontológica ${ }^{34}$.

As médias relativas aos indivíduos dos géneros masculino e feminino são semelhantes, não existindo diferenças estatisticamente significativas, levando assim a concluir que as atitudes éticas dos profissionais de saúde não são influenciáveis pelo género do profissional. O que parece sugerir uma diminuição da importância relativa da "ética do cuidado" face às outras correntes éticas, nomeadamente ao principialismo.

Na literatura consultada existe um estudo que aponta no sentido de que pode existir diferenças de atitude ética devidas ao género ${ }^{35}$ e outros estudos, tal como este, que referem que não existem diferenças ${ }^{29,36}$.

No presente estudo foi encontrada uma correlação positiva fraca na associação das atitudes éticas com a idade e o número de anos de profissão, concluindo-se que as atitudes éticas dos profissionais de saúde não são influenciáveis pela idade e número de anos de profissão. No entanto na literatura consultada foram vários os estudos encontrados a referir que existem diferenças de atitude ética devidas à idade $37,38,39$.

A média das pontuações foi superior no grupo dos profissionais que trabalham na sub-região de saúde de Viseu e inferior nos que trabalham na sub-região de Castelo Branco. Constatando-se, pelo presente estudo, que as atitudes éticas dos profissionais de saúde parecem ser mais firmes nas sub-regiões de saúde de Viseu e Aveiro e menos firmes nas sub-regiões de saúde de Leiria e Castelo Branco. Na ponderação deste facto deve-se ter em consideração o baixo número de respostas recebidas de Viseu.

Cada administração distrital dos serviços médico-sociais, criadas em 1974 em pleno processo revolucionário, desenvolveu-se com total autonomia, que foi alargada com a sua transformação em administrações regionais de saúde em $1982^{18}$, pelo que cada sub-região herdeira destas administrações tem uma história e cultura muito próprias, o que talvez explique as diferenças encontradas neste estudo.

A reflexão ética no âmbito das organizações implica a existência de certas condições, sendo que a principal é a própria "comunidade moral" da organização, isto é, o conjunto de normas morais comuns aos seus membros, porque as organizações mais não são do que a complexa articulação de interesses individuais que perseguem objetivos comuns. Mas devem fazê-lo respeitando a dignidade do cidadão e os seus direitos fundamentais ${ }^{40}$.

A cultura organizacional surge: quando existe uma partilha de valores e crenças; um quadro de referência da ação coletiva; e um sistema de ideias resultantes: da história da organização; da definição das situações pelos agentes dominantes; da interpretação cumulativa, por parte dos intervenientes; e do sentido das ações contínuas e recíprocas ${ }^{41}$.

A média das pontuações foi superior no grupo dos profissionais que declararam trabalhar em zona urbana (4.06) em relação aos que declararam trabalhar em zona rural ou semiurbana, e essa diferença foi estatisticamente significativa ( $p<0.001$ ), levando assim a concluir que as atitudes éticas dos profissionais de saúde são mais firmes nos que trabalham em área urbana.

Já o estudo anteriormente descrito de Barry Hoffmaster e colaboradores ${ }^{35}$ refere a existência de diferença nas 
atitudes éticas dos profissionais de saúde em função da área geográfica em que trabalham.

Considerando "mais firme" a obtenção de valores superiores na avaliação feita através da aplicação da Escala de atitudes perante os direitos à informação e ao consentimento, elaborada e validada por José Carlos Martins ${ }^{15}$, pode-se concluir, para os profissionais de saúde dos Centros de Saúde da Administração Regional de Saúde do Centro que responderam ao questionário, que as atitudes éticas dos profissionais de saúde perante os direitos à informação e ao consentimento dos pacientes:

A. Parecem ser mais firmes nos médicos que nos enfermeiros;

B. Não são influenciáveis pelo género;

C. Não são influenciáveis pela idade e número de anos de profissão;

D. Parecem ser mais firmes nos profissionais que trabalham nas sub-regiões de saúde de Viseu e Aveiro;

E. Parecem ser mais firmes nos que declaram trabalhar em área urbana.

\section{Referências Bibliográficas}

1. CORTINA, A. El quehacer ético. Guía para la educación moral. Madrid: Aula XXI/Santillana, 1996.

2. CORTINA, A. La ética de la sociedad civil. Madrid: ANAYA; 1994.

3. SAVATER, F. Invitación a la ética. Barcelona: Anagrama; 1986.

4. SAVATER, F. Ética para Amador. Barcelona: Ariel; 1992.

5. GRACIA, D. Fundamentos de bioética. Madrid: EUDEMA Universidad; 1989.

6. GRACIA, D. Planteamiento general de la bioética. In: GRACIA, D. Fundamentación y enseñanza de la bioética. Santa Fe de Bogotá: El Búho; 1998.

7. DÍAZ, B. et al. Necesidades de formación en bioética en la Comunidad de Madrid. Atencion Primaria. 2005; 35: 240-245.

8. ARCHER, L. Ainda os direitos do Homem. O consentimento informado. Brotéria. 1999; 148(2): 155-164.

9. VOLLMAN, J. Informed consent. A historical and medical perspective. In: OKASHA, A., ARBOLEDA-FLÓREZ, J., SARTORIUS, N. (eds.). Ethics, culture and psychiatry. Washington: American Psychiatric Press, 2000; 167-188.

10. MARTINS, JC. Atitudes dos doentes oncológicos perante os direitos à informação e ao consentimento. Revista de Investigação em Enfermagem. 2003; 8: 49-62.

11. MARTINS, JC. Os enfermeiros e os direitos dos doentes à informação e ao consentimento: Perceções, atitudes e opiniões. Revista Referência. 2004; 12:15-26.

12. SIMÕES, JA. et al. Os médicos de família e os direitos dos doentes à informação e ao consentimento. Revista Portuguesa de Bioética. 2007; 3:315-330.

\section{Agradecimentos}

A todos os profissionais dos centros de saúde da Administração Regional de Saúde do Centro que responderam ao questionário, aceitando fazer parte do presente estudo.

À Dra Denise Alexandre pela sua colaboração na análise estatística deste estudo.

13. DIRECÇÃO DE SERVIÇOS DE INFORMAÇÃO E ANÁLISE DDE. Centros de Saúde e Hospitais: Recursos e produção do SNS: Ano de 2005. Lisboa: Direcção-Geral da Saúde; 2006.

14. CARDOSO, SM. Notas epidemiológicas. Coimbra: Faculdade de Medicina - Instituto de Higiene e Medicina Social; 1998.

15. MARTINS, JC. Atitudes dos doentes oncológicos perante os direitos à informação e ao consentimento. Revista de Investigação em Enfermagem. 2003; 8: 49-62.

16. MARTINS, J. et al. Caracterização dos profissionais de saúde em Portugal. Parte I - Quantos somos e quem somos. Revista Portuguesa de Clínica Geral. 2003;19(5):513-17.

17. MACHADO, MCS. A feminização da medicina. Análise Social. 2003;38(166):127-37.

18. BISCAIA, AR. et al. Cuidados de Saúde Primários em Portugal. Reformar para novos sucessos. $2^{\mathrm{a}}$ edição revista e actualizada ed. Lisboa: Padrões Culturais Editora; 2008.

19. ROBILLARD, HM. et al. Ethical issues in primary health care: a survey of practitioners' perceptions. J Community Health. 1989 Spring;14(1):9-17.

20. UDEN, G. et al. Ethical reasoning in nurses' and physicians' stories about care episodes. J Adv Nurs. 1992 Sep;17(9):1028-34.

21. GRUNDSTEIN-AMADO, R. Differences in ethical decision-making processes among nurses and doctors. J Adv Nurs. 1992 Feb;17(2):129-37.

22. ROBERTSON, DW. Ethical theory, ethnography, and differences between doctors and nurses in approaches to patient care. J Med Ethics. 1996 Oct;22(5):292-9.

23. RICHTER, J. et al. Decision-making in the treatment of elderly people: a cross-cultural comparison between Swe- 
dish and German physicians and nurses. Scand J Caring Sci. 2002 Jun;16(2):149-56.

24. HARIHARAN, S. et al. Knowledge, attitudes and practice of healthcare ethics and law among doctors and nurses in Barbados. BMC Med Ethics. 2006;7:E7.

25. SIMÕES, JA. et al. Os médicos de família e os direitos dos doentes à informação e ao consentimento. Revista Portuguesa de Bioética. 2007; 3: 315-330.

26. SILVA, L. et al. Bioética e atenção básica: Um estudo exploratório dos problemas éticos vividos por enfermeiros e médicos no PSF. Cogitare Enfermagem. 2006;11(2):133-42.

27. RICKARD, M. et al. Caring and justice: a study of two approaches to health care ethics. Nurs Ethics. 1996 Sep;3(3):212-23.

28. KOLLEMORTEN, I. et al. Ethical aspects of clinical decision-making. J Med Ethics. 1981 Jun;7(2):67-9.

29. GRAMELSPACHER, GP. et al. Perceptions of ethical problems by nurses and doctors. Arch Intern Med. 1986 Mar;146(3):577-8.

30. GALLAGHER, A. Medical and nursing ethics: never the twain? Nurs Ethics. 1995 Jun;2(2):95-101.

31. WALKER, RM, et al. Physicians' and nurses' perceptions of ethics problems on general medical services. J Gen Intern Med. 1991 Sep-Oct;6(5):424-9.

32. OBERLE, K. et al. Doctors' and nurses' perceptions of ethical problems in end-of-life decisions. J Adv Nurs. 2001 Mar;33(6):707-15.

33. NUNES, R. Investigação e ensino da bioética em Portugal. In: Neves MdCP, editor. Comissões de ética: das bases teóricas à actividade quotidiana. $2^{\text {a }}$ edição revista e aumentada ed. Coimbra: Gráfica de Coimbra; 2002. p. 103-8.

34. NUNES, R. Ética, bioética e responsabilidade. Humanística e Teologia. 1997;18(2-3):279-90.

35. HOFFMASTER, CB. et al. Ethical decision making by family doctors in Canada, Britain and the United States. Soc Sci Med. 1991;33(6):647-53.

36. KOLLEMORTEN, I. et al. Ethical aspects of clinical decision-making. J Med Ethics. 1981 Jun;7(2):67-9.

37. CHRISTIE, RJ. et al. How family physicians approach ethical problems. J Fam Pract. 1983 Jun;16(6):1133-8.

38. BREMBERG, S. et al. Patients' autonomy and medical benefit: ethical reasoning among GPs. Fam Pract. 2000 Apr;17(2):124-8.

39. BITON, V. et al. The relationship between the application of the nursing ethical code and nurses' work satisfaction. Int J Nurs Pract. 2003 Jun;9(3):140-57.

40. BRANDÃO, C. A ética empresarial e os fundos socialmente responsáveis. Porto: Vida Económica; 2004.

41. NEVES, A. Gestão na administração pública. Alcabideche: Editora Pergaminho; 2002.

\section{Conflito de Interesses}

Os autores declaram não ter conflitos de interesse para o conteúdo deste artigo.

\section{Endereço para Correspondência:}

José Augusto Rodrigues Simões

USF Marquês de Marialva

Centro de Saúde de Cantanhede, Av. 25 de Abril, 44

3060-123 Cantanhede

Email: jarspt@sapo.pt 